\title{
Interacting Fock spaces and Gaussianization of probability measures
}

\author{
Luigi Accardi ${ }^{1}$ \\ Marek Bozejko ${ }^{2}$
}

\footnotetext{
${ }^{1}$ Graduate School of Polymathematics, Nagoya University, Centro V. Volterra Università di Roma Tor Vergata, Roma, Italy

${ }^{2}$ Instytut Matematyczny, Uniwersytet Wroclawski, Plac Grunwaldzki 2/4, 50-384 Wroclaw, Poland
} 


\section{Contents}

1 Introduction 3

2 Preliminaries on one mode interacting Fock spaces 4

3 Preliminaries on orthogonal polynomials: one variable 5

4 Gaussianization in one variable: symmetric measures 6

5 Gaussianization in one variable: arbitrary measures $\quad 7$

6 Universal convolution of probability measures 9

$\begin{array}{lll}7 & 2-d \text { file } & 10\end{array}$

8 Orthogonal polynomials in 2 variables 10

9 Multivariate Gaussianization $\quad 14$ 


\begin{abstract}
We prove that any probability measure on $\mathbb{R}$, with moments of all orders, is the vacuum distribution, in an appropriate interacting Fock space, of the field operator plus (in the non symmetric case) a function of the number operator. A corollary of this is that all the momenta of such a measure are expressible in terms of the Jacobi parameters, associated to its orthogonal polynomials, by means of diagrams involving only non crossing pair partitions (and singletons, in the non symmetric case). This means that, with our construction, the combinatorics of the momenta of any probability measure (with all moments) is reduced to that of a generalized Gaussian. This phenomenon we call Gaussianization. Finally we define, in terms of the Jacobi parameters, a new convolution among probability measures which we call universal because any probability measure (with all moments) is infinitely divisible with respect to this convolution. All these results are extended to the case of many (in fact infinitely many) variables.
\end{abstract}

\title{
1 Introduction
}

The notion of interacting Fock space emerged from the stochastic limit of QED [AcLu92] and was axiomatized in [AcLuVo97]. The zero space-time dimensional case corresponds to a non linear deformation of the usual (Boson, Fermion or $q$-deformed) one mode Fock spaces. On the other hand, in [BoWy97] have introduced a new convolution among probability measures on $\mathbb{R}$, with all moments, generalizing the free convolution and based on Szëgo's theorem which associates a continuous fraction to any such a measure.

By combining the two techniques it is possible to construct a canonical isomorphism between the $L^{2}$-space associated to any symmetric probability measure on $\mathbb{R}$ with all moments and an appropriate one-mode interacting Fock space, in which the number vectors correspond to the orthogonal polynomials for this measure and the the field operator to the position operator. All the one mode interacting Fock spaces are shown to arise in this way.

Moreover, by adding a function of the number operator to the field operator one obtains a symmetric operator whose momenta, with respect to the vacuum vector, coincide with the momenta of a non necessarily symmetric probability measure on $\mathbb{R}$ with all moments. All such measures can be obtained with this construction.

The construction can be extended to the multidimensional (even infinite 
dimensional) case and provides a new natural technique to deal with orthogonal polynomials.

These results strongly support the conjecture that the interacting Fock space provides a natural frame to deal with non Gaussian measures, i.e. with interacting fields.

\section{Preliminaries on one mode interacting Fock spaces}

An interacting Fock space is a Hilbert space associated to an arbitrary (real or complex) pre-Hilbert space $\mathcal{K}$ through a procedure, motivated by the stochastic limit of quantum electrodynamics, and which is a generalization of the known second quantizations functors (free, $q$-deformations, ...). In the present paper we shall only consider the case in which $\mathcal{K}$ is the complex numbers which, in physical language, corresponds to a one mode field (equivalently a field with a 1-particle space of zero space-time dimensions). For the general case we refer to [AcLuVo97].

Definition 1 Let $\mathcal{K}$ be a one-dimensional complex Hilbert space. Then, for each $n \in \mathbb{N}$ also $\mathcal{K}_{n}:=\otimes^{n} \mathcal{K}$ is 1-dimensional, and we identify it to the multiples of a non-normalized vector denoted $a^{+n} \Phi$. With this identification, a pre-scalar products $(\cdot \mid \cdot)_{n}$ can only have the form

$$
(z \mid w)_{n}:=\lambda_{n}(z, w) \bar{z} w \quad ; \quad z, w \in \mathbb{C}
$$

where the $\lambda_{n}$ are positive numbers. The one mode interacting Fock space $\Gamma\left(\mathbb{C} ;\left(\lambda_{n}\right)\right)$ is the Hilbert space obtained by completing the orthogonal sum $\bigoplus_{n}\left\{\mathcal{K}_{n},(\cdot \mid \cdot)_{n}\right\}$ where the scalar product $(\cdot \mid \cdot)_{n}$ is given by (1) and the sequence $\left(\lambda_{n}\right)$ satisfies the following condition

$$
\lambda_{n}=0 \Longrightarrow \lambda_{N}=0 \quad ; \quad \forall N \geq n
$$

Under this condition, the creator

$$
a^{+}: a^{+n} \Phi \rightarrow a^{+(n+1)} \Phi
$$

is well defined and, with the convenction that $0 / 0=0$, the annihilator a is given by the rule:

$$
a: a^{+(n+1)} \Phi \rightarrow \frac{\lambda_{n+1}}{\lambda_{n}} a^{+n} \Phi
$$


which is equivalent to the commutation relation

$$
a a^{+}=\frac{\lambda_{N+1}}{\lambda_{N}}
$$

where the number operator $N$ is defined in the usual way:

$$
N\left(x_{1} \otimes \ldots \otimes x_{n}\right)=n\left(x_{1} \otimes \ldots \otimes x_{n}\right) \quad ; \quad n \in \mathbb{N}
$$

and the right hand side of (4) is uniquely defined by the spectral theorem The algebraic relation (5), together with the condition

$$
a \Phi=0
$$

uniquely characterizes the interacting Fock space structure. The resulting Hilbert space is finite dimensional if and only if $\lambda_{n}=0$ for some $n$.

\section{Preliminaries on orthogonal polynomials: one variable}

The following theorem is well known [Szë38]

Theorem 1 Let $\mu$ be a probability measure on $\mathbb{R}$ with finite moments of all orders. There exists two sequences of real numbers $\alpha_{n} \in \mathbb{R}, \omega_{n} \geq 0$, such that, denoting $\left\{P_{n}\right\}$ the sequence of orthogonal polynomials associated to $\mu$, normalized so that $P_{-1}=0, P_{0}=1, P_{1}(x)=x-\alpha_{1}$, the following relations hold for each $n \in \mathbb{N}$ :

$$
\begin{gathered}
\left(x-\alpha_{n+1}\right) P_{n}(x)=P_{n+1}(x)+\omega_{n} P_{n-1}(x) \\
\int P_{n}(x) P_{m}(x) d \mu(x)=\delta_{n, m} \omega_{1} \omega_{2} \ldots \omega_{n}
\end{gathered}
$$

Moreover $\mu$ is symmetric if and only if $\alpha_{n}=0$ for each $n$ and $\mu$ is supported in a finite number of points if and only if $\omega_{n}=0$ for some $n$.

Proof. A standard induction procedure.

Remark. The sequences $\left(\omega_{n}\right)\left(\alpha_{n}\right)$, defined by the previous theorem, shall be called the Jacobi parameters (or sequences) associated to $\mu$. 


\section{Gaussianization in one variable: symmetric measures}

Theorem 2 Let $\mu$ be a symmetric probability measure on $\mathbb{R}$ with all moments and let $\left(P_{n}\right)$ be the associated sequence of orthogonal polynomialys in $L^{2}(\mathbb{R}, \mu)$. Then there exists a unique one mode interacting Fock space $\Gamma\left(\mathbb{C} ;\left(\lambda_{n}\right)\right)$ and a unitary isomorphism

$$
U: \Gamma\left(\mathbb{C} ;\left(\lambda_{n}\right)\right) \rightarrow L^{2}(\mathbb{R}, \mu)
$$

with the following properties:

$$
\begin{gathered}
U \Phi=1 \\
U a^{+} U^{*} P_{n}=P_{n+1} \\
U\left(a^{+}+a\right) U^{*}=Q
\end{gathered}
$$

where $Q$ is multiplication by $x$ in $L^{2}(\mathbb{R}, \mu)$. Conversely, given a one mode interacting Fock space the vacuum distribution $\mu$ of any self-adjoint extension of $a^{+}+a$ is such that the interacting Fock space, associated to $\mu$ with the construction of the first part of the theorem is the original one.

Proof. Let $\left(\omega_{n}\right)$ be the Jacobi sequence associated to $\mu$ and suppose first that

$$
\omega_{n}>0 ; \quad \forall n
$$

Define the sequence $\left(\lambda_{n}\right)$ by

$$
\lambda_{1}=1 \quad ; \quad \omega_{n+1}=\frac{\lambda_{n+1}}{\lambda_{n}}
$$

Then there exists a one mode interacting Fock space characterized by the condition

$$
a a^{+}=\omega_{N+1}=\frac{\lambda_{N+1}}{\lambda_{N}}
$$

The map

$$
a^{+n} \Phi \in \Gamma\left(\mathbb{C},\left(\lambda_{n}\right)\right) \mapsto P_{n} \in L^{2}(\mathbb{R}, \mu)
$$

preserves orthogonality by construction and since

$$
\left\|a^{+n} \Phi\right\|^{2}=\left(a^{+(n-1)} \phi \mid a a^{+} a^{+(n-1)} \Phi\right)_{n-1}=
$$




$$
=\omega_{n}\left\|a^{+(n-1)} \Phi\right\|^{2}=\ldots=\omega_{n} \cdot \omega_{n-1} \cdot \ldots \cdot \omega_{1}=\lambda_{n}
$$

it preserves norms. Therefore it has a unique unitary extension $U$. Now let $t \in \mathbb{R}$ and consider, for any $\Psi \in \Gamma\left(\mathbb{C} ;\left(\lambda_{n}\right)\right)$ and for any $n \in \mathbb{N}$ :

$$
\begin{gathered}
\left(\Psi \mid\left(a^{+}+t a\right) a^{+n} \Phi\right)=\left(\Psi \mid a^{+n+1} \Phi\right)+t\left(\Psi \mid a a^{+} a^{+(n-1)} \Phi\right)= \\
=\left\langle U \Psi, P_{n+1}\right\rangle_{L^{2}(\mathbb{R}, \mu)}+t \omega_{n}\left\langle U \Psi, P_{n-1}\right\rangle_{L^{2}(\mathbb{R}, \mu)}
\end{gathered}
$$

For $t=0$, this gives (??). For $t=1$, by the Szëgo induction relation (??) for the orthogonal polynomials the right hand side of (15) becomes

$$
\left\langle U \Psi, x P_{n}\right\rangle_{L^{2}(\mathbb{R}, \mu)}=\left(\Psi \mid U^{*} x U a^{+n} \Phi\right)
$$

which is equivalent to (4).

It remains to be discussed the case when $\lambda_{n}$ is zero starting from some $n$. If there exists an $n$ such that $\omega_{n}=0$, then $P_{n}(x)=0 \mu$ a.e. and therefore by (3.1) also $P_{n+1}(x)=0, \mu$-a.e. Therefore, by induction all $P_{n+k}$ are zero, for $k \geq 0$. Thus $L^{2}(\mathbb{R}, \mu)$ is finite dimensional and this can only be if $\mu$ has support in a finite set of points.

\section{Gaussianization in one variable: arbitrary measures}

In this section we extend to the non symmetric case a result of [AcLuVo97] for the calculation of vacuum momenta of the field operator in a interacting Fock space. This allows, given an arbitrary probability measure $\mu$ with all moments, to express its moments through its Jacobi parameters $\omega_{n}, \alpha_{n}$.

Let $\mathcal{H}$ be a real Hilbert space. For $\omega_{n}$ and $\alpha_{n}$ as above, we introduce the interacting Fock space $\mathcal{F}(\mathcal{H})=\oplus \mathcal{H}^{\otimes n}$ with creation and annihilation operators $a(f), a^{+}(f)(f \in \mathcal{H})$ characterized by the relations

$$
\begin{gathered}
a^{+}(f) x_{1} \otimes \ldots \otimes x_{n}=f \otimes x_{1} \otimes \ldots \otimes_{n}, \quad x_{j} \in \mathcal{H} \\
a(f) a^{+}(g)=\omega_{N+1}\langle f, g\rangle \\
a(f) \Omega=0
\end{gathered}
$$

It is easy to verify that the axioms of the interacting Fock spaces are fulfilled and, for each natural integer $n$, the scalar product on the $n$-th particle space is uniquely defined by the above relations and one has

$$
\left\|x_{1} \otimes \ldots \otimes x_{n}\right\|_{\omega}^{2}=\lambda_{n}\left\|x_{1}\right\|^{2} \ldots\left\|x_{n}\right\|^{2}
$$


where $\lambda_{n}=\omega_{n} \ldots \omega_{2} \omega_{1}$. Then the operator $\alpha_{N}$, where the number operator $N$ is defined by (2.5), is uniquely defined by the spectral theorem and one has

$$
\alpha_{N}\left(x_{1} \otimes \ldots \otimes x_{n}\right)=\alpha_{n}\left(x_{1} \otimes \ldots \otimes x_{n}\right)
$$

With these notations the following theorem holds:

Theorem 3 The operator

$$
T_{\mu}(f)=a(f)+a^{+}(f)+\alpha_{N}
$$

is symmetric, and for any natural integer $n$ and $f_{j} \in \mathcal{H}(j=1, \ldots, n)$

$$
\begin{gathered}
\left\langle T\left(f_{1}\right) T\left(f_{2}\right) \ldots T\left(f_{n}\right) \Omega \mid \Omega\right\rangle= \\
\sum_{\vartheta=\left(B_{1}, \ldots, B_{m}, B_{m+1}, B_{k}\right) \in \overline{\mathcal{P}}_{2}(1, \ldots, n)}\left\langle f_{k_{1}}, f_{l_{1}}\right\rangle \ldots\left\langle f_{k_{m}}, f_{l_{m}}\right\rangle t_{\mu}(\vartheta)
\end{gathered}
$$

where $\vartheta$ is non-crossing partition of the set $\{1,2, \ldots, n\}$, with blocks $\left|B_{j}\right| \leq 2$, $j=1, \ldots, k$ and

$$
B_{1}=\left\{k_{1}, l_{1}\right\}, \ldots, B_{m}=\left\{k_{m}, l_{m}\right\}
$$

while $\left|B_{j}\right|=1$ for $j \geq m+1$. The function $t_{\mu}(\vartheta)$ has the following form:

$$
t_{\mu}(\vartheta)=\prod_{\substack{B_{j} \in \vartheta \\\left|B_{j}\right|=2}} \omega\left(d_{\vartheta}\left(B_{j}\right)\right) \prod_{\substack{B_{k} \in \vartheta \\\left|B_{k}\right|=1}} \alpha\left(d_{\vartheta}\left(B_{k}\right)\right)
$$

where $d_{\vartheta}\left(B_{j}\right)$ denote the depth of the block $B_{j}$ in the partition $\vartheta$ defined, as in [AcLuVo97], by

$$
d_{\vartheta}\left(B_{j}\right):=\left|\left\{k: B_{j} \subset B_{k} \in \vartheta\right\}\right|
$$

and $|\cdot|$ denotes cardinality.

Proof. The proof is just verification of the formula (1).

As a corollary we get the following interesting formula, relating the Jacobi parameter of a measure $\mu$ with its moments.

Corollary (2). If $\mu$ is a probability measure on $\mathbb{R}$ with all moments, then

$$
\mu_{n}(\mu)=\int x^{n} d \mu=\sum_{\vartheta \in \mathcal{P}_{2}(1, \ldots, n)} t_{\mu}(\vartheta)
$$


Theorem 4 Let $\mu$ be a probability measure on $\mathbb{R}$ with all moments, let $\left(P_{n}\right)$ be the associated sequence of orthogonal polynomialys in $L^{2}(\mathbb{R}, \mu)$ and let $\left(\omega_{n}\right),\left(\alpha_{n}\right)$ be the Jacobi parameters of $\mu$. Then there exists a unique one mode interacting Fock space $\Gamma\left(\mathbb{C} ;\left(\lambda_{n}\right)\right)$ and a unitary isomorphism

$$
U: \Gamma\left(\mathbb{C} ;\left(\lambda_{n}\right)\right) \rightarrow L^{2}(\mathbb{R}, \mu)
$$

with the following properties:

$$
\begin{gathered}
U \Phi=1 \\
U a^{+} U^{*} P_{n}=P_{n+1} \\
U\left(a^{+}+a+\alpha_{N}\right) U^{*}=Q
\end{gathered}
$$

where $Q$ is multiplication by $x, N$ is the number operator defined in (2.5) and $\alpha_{N}$ is defined by the spectral theorem.

Conversely given a one mode interacting Fock space the vacuum distribution $\mu$ of any self-adjoint extension of $a^{+}+a$ is such that the interacting Fock space associated to $\mu$ is the original one.

Proof. The proof is similar to the one of Theorem just using the induction relation (??) for non symmetric measures.

\section{Universal convolution of probability mea- sures}

The results of the previous sections suggest a natural notion of convolution among arbitrary probability measures with all moments.

Let $\mu_{1}, \mu_{2}$ be probability measures on $\mathbb{R}$ with all moments. Let, for $k=0,1$ and $n=0,1,2 \ldots,\left(P_{n}^{(k)}\right)$ be the orthogonal polynomials associated to $\mu_{k}$ and $\alpha_{n}^{(k)} \in \mathbb{R}, \omega_{n}^{(k)}>0$ be the corresponding Jacobi parameters. Then we know that

$$
\left(x-\alpha_{n}^{(k)}\right) P_{n}^{(k)}=P_{n+1}^{(k)}+\omega_{n}^{(k)} P_{n-1}^{(k)}
$$

and vice versa, each sequence $\alpha_{j}^{(k)} \in \mathbb{R}, \omega_{j}^{(k)}>0$ give a unique state on the *-algebra $\mathbb{C}[x]$ of polynomials in one real variable.

Definition 2 The universal convolution of $\mu_{1}$ and $\mu_{2}$ is the unique state $\mu:=$ $\mu_{1} \times \mu_{2}$, on $\mathbb{C}[x]$, whose Jacobi parameters $\alpha_{n}(\mu), \omega_{n}(\mu)$ are characterized by:

$$
\alpha_{n}(\mu)=\alpha_{n}^{(1)}\left(\mu_{1}\right)+\alpha_{n}^{(2)}\left(\mu_{2}\right)
$$




$$
\omega_{n}(\mu)=\omega_{n}^{(1)}\left(\mu_{1}\right)+\omega_{n}^{(2)}\left(\mu_{2}\right)
$$

Theorem 5 The universal convolution has the following properties:

(1) associativity (2) commutativity (3) positivity, i.e.: if $\mu_{1}, \mu_{2}$ are probability measure, then $\mu_{1} \times \mu_{2}$ state on $\mathbb{C}[x]$. (4) For a dilation $D_{\lambda} \mu(E)=\mu\left(\lambda^{-1} E\right)$, $E \subset \mathbb{R}$ borel subset, $\lambda>0$, we have

$$
D_{\lambda}\left(\mu_{1} \times \mu_{2}\right)=\left(D_{\lambda} \mu_{1}\right) \times\left(D_{\lambda} \mu_{2}\right)
$$

(5) For each symmetric measure $\mu$, and $N=1,2, \ldots$

$$
S_{N}(\mu)=\left(D \frac{1}{\sqrt{N}} \mu\right) \times\left(D \frac{1}{\sqrt{N}} \mu\right) \times \ldots \times\left(D \frac{1}{\sqrt{N}} \mu\right)=\mu
$$

If $\delta_{x}$-denote the point mass of $x \in \mathbb{R}$, then $\left(\delta_{x}\right) \times \mu=\delta_{x} * \mu_{1}$ where $*$ is the classical convolution.

Hence $S_{N}(\mu)=\mu$, therefore in the central limit theorem each measure can appears and each measure is infinitely divisible for this convolution.

$$
\int P_{n}^{2} d \mu=\omega_{1} \omega_{2} \ldots \omega_{n}
$$

Therefore for each measure $\mu$

$$
\begin{aligned}
& \omega_{n}\left(D_{\lambda} \mu\right)=\lambda^{2} \omega_{n}(\mu) \\
& \alpha_{n}\left(D_{\lambda} \mu\right)=\lambda \alpha_{n}(\mu)
\end{aligned}
$$

when for the measure $\mu$

$$
\left(x-\alpha_{n}(\mu)\right) P_{n}=P_{n+1}+\omega_{n}(\mu) P_{n-1}
$$

Therefore $\omega_{n}\left(S_{N}(\mu)\right)=N \cdot \omega_{n}\left(D \frac{1}{\sqrt{N}}(\mu)\right)=\omega_{n}(\mu)$ since $\alpha_{n}(\mu)=0$, therefore $S_{N}(\mu)=\mu$. The other properties are easy to check.

\section{$7 \quad 2-d$ file}

\section{Orthogonal polynomials in 2 variables}

Let $\mu$ be a probability measure on $\mathbb{R}^{2}$ with all moments and let $P_{k, n}$ denote the associated orthogonal polynomials. Then the following theorem holds: 
Proposition 1 Under the above assumptions there exists sequences $\alpha_{k, l}^{(i)} \in \mathbb{R}$, $\omega_{k, l}^{(i)}>0, i=1,2$ such that:

$$
\begin{aligned}
& x_{1} P_{k, l}=P_{k+1, l}+\alpha_{k, l}^{(1)} P_{k, l}+\omega_{k, l}^{(1)} P_{k-1, l} \\
& x_{2} P_{k, l}=P_{k, l+1}+\alpha_{k, l}^{(2)} P_{k, l}+\omega_{k, l}^{(2)} P_{k, l-1}
\end{aligned}
$$

Moreover

$$
\int P_{k, l}^{2} d \mu=\omega\left(\gamma_{k, l}\right)
$$

where $\gamma_{k, l}$ is any geodesic path from $(0,0)$ to $(k, l)$ on the lattice $\mathbb{Z} \oplus \mathbb{Z}$ and if

$$
\gamma_{k, l}=\left(g_{1}, g_{2}, \ldots, g_{k-1}\right)
$$

then

$$
\omega\left(\gamma_{k, 1}\right)=\prod_{j=1}^{k+l} \omega\left(g_{j}\right)
$$

where

$$
\omega\left(g_{j}\right)=\omega_{s, t}^{(\varepsilon)}, \quad \varepsilon=1,2 \quad(s, t)=g_{1} g_{2} \ldots g_{j}
$$

as a vector on $\mathbb{Z} \oplus \mathbb{Z}$ and $\varepsilon$ depends on the geodesic path. Finally $\mu$ is symmetric if and only if all the coefficients $\alpha_{k, l}^{(1)}, \alpha_{k, l}^{(2)}$ are zero.

Proof. The result is known but we give a quantum proof of it. We begin to apply the Gram-Schmidt orthogonalization in $L^{2}\left(\mathbb{R}^{2}, \mu\right)$ to the monomials $\left\{x_{1}^{n} x_{2}^{m}\right\}$ and we normalize the orthogonal polynomials so that the highest coefficient is equal 1. Since the $P_{k, l}$ are independent

$$
x_{1} P_{k, l}=P_{k+1, l}+\sum_{i+j \leq k+l} \alpha_{i j} P_{i, j}
$$

we claim that, if $i+j<k+l-1$, then $\alpha_{i, j} \equiv 0$. This follows from orthogonality property of $P_{i, j}$. To get formula (2) we use the relation (1) and induction

$$
\begin{gathered}
\int P_{k+1, l}^{2}=\int P_{k+1, l}\left(x_{1} P_{k, l}-\alpha_{k, l}^{(1)} P_{k, l}-\omega_{k, l}^{(1)} P_{k-1, l}\right) d \mu= \\
=\int x_{1} P_{k+1, l} P_{k, l} d \mu=\int\left(P_{k+2, l}+\alpha_{k+1, l}^{(1)} P_{k+1, l}+\omega_{k+1, l}^{(1)} P_{k, l}\right) P_{k, l}=
\end{gathered}
$$




$$
=\omega_{k+1, l}^{(1)} \int P_{k, l}^{2} d \mu=\prod_{j=1}^{k+l} \omega_{y_{j}}^{\left(\varepsilon_{i}\right)}
$$

Now we prove that $\mu$ is symmetric if and only if all the coefficients $\alpha_{k, l}^{(1)}, \alpha_{k, l}^{(2)}$ are zero.

Now denote $a_{j}^{+}(j=1,2)$ the creation operators

$$
a_{1}^{+} P_{k, l}=P_{k+1, l} \quad ; \quad a_{2}^{+} P_{k, l}=P_{k, l+1} \quad ; \quad j=1,2
$$

One easily sees that these are well defined and have densely defined adjoints $a_{j}(j=1,2)$, called annihilation operators. Moreover

$$
\left[a_{\alpha}^{+}, a_{\beta}^{+}\right]=\left[a_{\alpha}, a_{\beta}\right]=0 \quad, \alpha \neq \beta
$$

and, denoting $\Phi$ the constant function 1, one has

$$
a_{1}^{+k} a_{2}^{+n} \Phi=P_{k, n}
$$

Introducing the number operators $N_{1}, N_{2}$, defined by

$$
N_{1} P_{k, n}:=k P_{k, n} \quad, \quad N_{2} P_{k, n}:=n P_{k, n}
$$

one easily verifies the relations

$$
a_{1} \Phi=a_{2} \Phi=0
$$

Suppose first that $\mu$ is a symmetric measure. Then one easily sees that

$$
\begin{aligned}
& a_{1} P_{k, l}=\omega_{k, l}^{(1)} P_{k-1, l} \\
& a_{2} P_{k, l}=\omega_{k, l}^{(2)} P_{k, l-1}
\end{aligned}
$$

From this one deduces

$$
\begin{gathered}
a_{\alpha} a_{\alpha}^{+}=\omega_{N_{1}, N_{2}}^{(\alpha)} ; \quad \alpha=1,2 \\
a_{1} a_{2}^{+}=\frac{\omega_{N_{1}, N_{2}+1}^{(1)}}{\omega_{N_{1}, N_{2}}^{(1)}} a_{2}^{+} a_{1}
\end{gathered}
$$

in fact

$$
a_{1} a_{2}^{+} P_{k, l}=a_{1} P_{k, l+1}=\omega_{k, l+1}^{(1)} P_{k-1, l+1}
$$




$$
=\left(\frac{\omega_{k, l}}{\omega_{k_{l}}}\right) \omega_{k, l}^{(1)} P_{k-1, l+1}=a_{2}^{+} a_{1} P_{k, l}
$$

Similarly

$$
a_{2} a_{1}^{+}=\frac{\omega_{N_{1}+1, N_{2}}^{(2)}}{\omega_{N_{1}, N_{2}}^{(2)}} a_{1}^{+} a_{2}
$$

in fact

$$
a_{2} a_{1}^{+} P_{k, l}=a_{2} P_{k+1, l}=\omega_{k+1, l}^{(2)} P_{k+1, l-1}=\left(\frac{\omega_{k+1, l}^{(2)}}{\omega_{k, l}^{(2)}}\right) \omega_{k l}^{(2)} P_{k+1, l-1}
$$

Moreover

$$
\begin{gathered}
a_{2}^{+} a_{1} P_{k, l}=\omega_{k, l}^{(1)} a_{2}^{+} P_{k-1, l}=\omega_{k, l}^{(1)} P_{k-1, l+1} \\
a_{1}^{+} a_{2} P_{k l}=\omega_{k, l}^{(2) k l} a_{1}^{+} P_{k_{1} l-1}=\omega_{k l}^{(2)} P_{k+1, l-1}
\end{gathered}
$$

But taking the adjoint of (2) we obtain

$$
a_{2} a_{1}^{+}=\omega_{N_{1}, N_{2}+}^{(1)} / \omega_{N_{1}, N_{2}}^{(1)} a_{1}^{+} a_{2}
$$

from this we deduce the cohomological relation

$$
\frac{\omega_{N_{1}, N_{2}+1}^{(2)}}{\omega_{N_{1}, N_{2}}^{(1)}}=\frac{\omega_{N_{1}+1, N_{2}}^{(2)}}{\omega_{N_{1}, N_{2}}^{(2)}}
$$

Now denote $Q_{j}$ the multiplication by the $j$-th coordinate in $L^{2}\left(\mathbb{R}^{2}, \mu\right)$

$$
Q_{j} f\left(x_{1}, x_{2}\right):=x_{j} f\left(x_{1}, x_{2}\right) \quad ; \quad j=1,2
$$

From the induction relation we deduce

$$
Q_{j}=a_{j}^{+}+a_{j}
$$

But, since the position operators commute $\left(\left[Q_{1}, Q_{2}\right]=0\right)$ one should also have

$$
a_{1}^{+} a_{2}+a_{1} a_{2}^{+}=a_{2}^{+} a_{1}+a_{2} a_{1}^{+}
$$

and the commutativity condition (5) is equivalent to

$$
a_{1}^{+} a_{2}-a_{1}^{+} a_{1}=a_{2} a_{1}^{+}-a_{1} a_{2}^{+}
$$


equivalently, because of (2), (??)

$$
a_{1}^{+} a_{2}-a_{2}^{+} a_{2}=\frac{\omega_{N_{1}+1, N_{2}}^{(2)}}{\omega_{N_{1}, N_{2}}^{(2)}} a_{1}^{+} a_{2}-\frac{\omega_{N_{1}, N_{2}+1}^{(1)}}{\omega_{N_{1}, N_{2}}^{(1)}} a_{2}^{+} a_{1}
$$

but this is equivalent to

$$
\left(\frac{\omega_{N_{1}+1, N_{2}}^{(2)}}{\omega_{N_{1}, N_{2}}^{(2)}}-1\right) a_{1}^{+} a_{2}=\left(\frac{\omega_{N_{1}, N_{2}+1}^{(1)}}{\omega_{N_{1}, N_{2}}^{(1)}}-1\right) a_{2}^{+} a_{1}
$$

Now, because of the cohomological relation, the operators in parentheses are equal, so, if they where not zero one should have

$$
a_{1}^{+} a_{2}=a_{2}^{+} a_{1}
$$

which is evidently false. But this means that $\omega_{N_{1}, N_{2}}^{(2)}$ depends only on $N_{2}$ and $\omega_{N_{1}, N_{2}}^{(1)}$ only on $N_{1}$. Therefore the relations () become

$$
\begin{aligned}
& x_{1} P_{k, m}=P_{k+1, m}+\omega_{k}^{(1)} P_{k-1, m} \\
& x_{2} P_{k, m}=P_{k, m+1}+\omega_{m}^{(2)} P_{k, m-1}
\end{aligned}
$$

with

$$
P_{0,0} \equiv 1, \quad P_{1,0}\left(x_{1}, x_{2}\right)=x_{1}, \quad P_{0,1}\left(x_{1}, x_{2}\right)=x_{2}
$$

Lemma 1 For a symmetric probability measure $\mu\left(x_{1}, x_{2}\right)$ on $\mathbb{R}^{2}$ one has

$$
\int P_{k, m}^{2} d \mu=\prod_{j=1}^{k} \omega_{j}^{(1)} \prod_{l=1}^{m} \omega_{l}^{(2)}
$$

Proof. A simple induction argument.

\section{Multivariate Gaussianization}

Theorem 6 Let $\mathcal{P}\left(\mathbb{R}^{2}\right)$ be the set of symmetric probability measures on $\mathbb{R}^{2}$ with finite moments of all orders. Let $P_{n, m}\left(x_{1}, x_{2}\right)$ be the orthogonal polynomials in $L^{2}\left(\mathbb{R}^{2}, \mu\right)$. Then there exist two interacting Fock spaces $\Gamma_{1}, \Gamma_{2}$ and a unitary isomorphism

$$
U: \mathcal{H}:=\Gamma_{1} \otimes \Gamma_{2} \rightarrow L^{2}\left(\mathbb{R}^{n}, \mu\right)
$$


such that, denoting $Q_{j}$ the multiplication by the $j$-th coordinate in $L^{2}\left(\mathbb{R}^{2}, \mu\right)$ and denoting $a_{1}^{+}, a_{1}$ (resp. $a_{2}^{+}, a_{2}$ ) the creator and annihilator on $\Gamma_{1}$ (resp. $\left.\Gamma_{2}\right)$ one has

$$
\begin{aligned}
& U^{*} Q_{1} U=a_{1}^{+} \otimes 1+a_{1} \otimes 1 \\
& U^{*} Q_{2} U=1 \otimes a_{2}^{+}+1 \otimes a_{2}
\end{aligned}
$$

Proof. According to Theorem (x.), the inductive relations for the orthogonal polynomials in $L^{2}\left(\mathbb{R}^{2}, \mu\right)$ are

$$
\begin{aligned}
& x_{1} P_{k, n}=P_{k+1, n}+\omega_{k}^{(1)} P_{k-1, n} \\
& x_{2} P_{k, n}=P_{k, n+1}+\omega_{n}^{(2)} P_{k, n-1}
\end{aligned}
$$

Consider two 1-mode interacting Fock space $\Gamma_{1}, \Gamma_{2}$ corresponding to the sequences $\left(\omega_{n}^{(1)}\right),\left(\omega_{n}^{(2)}\right)$ respectively. Then defining

$$
x_{j}=a_{j}^{+}+a_{j} ; \quad j=1,2
$$

the sequence $a_{1}^{+k} a_{2}^{+n}$ satisfies

$$
\begin{gathered}
x_{1} a_{1}^{+k} a_{2}^{+n}=a_{1}^{+k+1} a_{2}^{+n}+\lambda_{k}^{(1)} a_{1}^{+k-1} a_{2}^{n} \\
x_{2} a_{1}^{+k} a_{2}^{+n}=a_{1}^{+k} a_{2}^{+n+1}+\lambda_{n}^{(2)} a_{1}^{+k} a_{2}^{+n+1} \\
\left\|a_{1}^{+k} a_{2}^{+n} \Phi\right\|^{2}=\left\|a_{1}^{+k} \Phi_{1}\right\| \cdot\left\|a_{2}^{+n} \Phi_{2}\right\|=\omega_{k}^{(1)} \ldots \omega_{1}^{(1)} \cdot \omega_{n}^{(2)} \ldots \omega_{1}^{(2)} \\
=\int P_{k, n}^{2}(x) \mu(d x)
\end{gathered}
$$

From this the statement easily follows.

Remark. The extension to the multidimensional case (and non symmetric) is now clear: one simply considers the incomplete countable tensor product of a sequence of interacting Fock spaces defined by the analogue of the relations (1), (2). The details shall be discussed elsewhere.

\section{References}

[1] [AcLu92]Accardi L., Lu Y.G.: Accardi L., Lu Y.G.: The Wigner Semicircle Law in Quantum Electro Dynamics. Commun. Math. Phys., 180 (1996), 605-632. Volterra preprint N.126 (1992) 
[2] [AcLuVo97] Accardi L., Lu Y.G., I. Volovich: Interacting Fock spaces and Hilbert module extensions of the Heisenberg commutation relations. Publications of IIAS (Kyoto) (1997)

[3] [Aho98a] L. Accardi, Y. Hashimoto and N. Obata Notions of Independence Related to the Free Group to appear in Infinite dimensional analysis quantum probability and related topics, N. 2 (1998) Volterra Preprint (1998) N. 311

[4] [Aho98b] L. Accardi, Y. Hashimoto and N. Obata Singleton Independence. In: Proceedings of the Quantum Probability Conference, Gdansk, July 1997 Publications of the Banach Center (1998) Volterra Preprint (1998) N. 310

[5] [BoLeiSp94] Bozejko M., Leinert M., Speicher R.: Convolution and limit theorems for conditionally free random variables. Preprint 1994, Pac. J. Math.

[6] [BoWy97] Bozeiko and Wysoczanski, New convolutions of measures and central limit theorem. Preprint Wroclaw (1997), p. 1-10.

[7] [Lu95c] Lu Y.G.: The interacting Free Fock Space and the Deformed Wigner Law. Preprint of Centro Volterra (1995), Nagoya J. of Math. vol.145, pp 1-29 (1997).

[8] [Lu96] Lu Y.G.: An interacting free Fock space and the reciprocal Wigner law. Preprint of Centro Volterra (1996), Probability and mathematical statistics, vol. 17, fasc. 1 (1997)

[9] [Lu97] Lu Y.G.: Anderson type interacting free Fock space, to appear in Inf. dim. an., quantum prob. and rel. topics (IDA-QP), N 2 (1998).

[10] [Sue85]Suetin, Orthogonal polynomials in 2 variables. Moscow (1985), (in russian).

[11] [Sze38] Szëgo, Orthogonal polynomials. (1938).

[12] [Kold91]Koldobskii A.L.: Schoenberg's problem on positive definite functions, Preprint January (1991) 